\title{
Envejecer: Una reflexión de la terapia ocupacional sobre el tiempo libre del anciano jubilado de sexo masculino no institucionalizado $(*)$
}

Alessandra Karina Salles Santos, Vanessa Cristina Ribeiro Gomes

\section{Resumen}

El objetivo de nuestra investigación es analizar lo que los ancianos jubilados de sexo masculino realizan en su tiempo libre y si están insertados en grupos de Tercera Edad - Asociaciones de Jubilados. Los sujetos de nuestra investigación son ocho ancianos hombres de sesenta a noventa años, frecuentadores de la Plaza Maria Olinda de Castro, localizada en el barrio Vila Progresso, en la ciudad de Sorocaba. Utilizamos como metodología la investigación cualitativa por ésta tener un carácter multidimensional y colocar al investigador en contacto directo con la clientela investigada. La recolección de datos fue realizada por medio de ocho entrevistas semiestructuradas, con una duración media de 30 minutos cada una. También se recolectó datos a través de fotos. Los resultados fueron divididos en cinco categorías: edad, estado civil, participantes o no de Grupos de Tercera Edad o Asociaciones de Jubilados, si poseen o no tiempo libre y en cuanto a los deseos de esos ancianos.

Pudimos verificar que los ancianos realizan actividades socio-recreativas, con eso se produce una disminución del estrés, aumento de la satisfacción personal y una interacción social más amplia donde el compartir es visto como algo muy importante. Por lo tanto, podemos considerar que esos ancianos están socializados, ejerciendo un papel activo en la comunidad y sociedad.

PALABRAS CLAVE: Envejecimiento, Terapia Ocupacional, Tiempo Libre, Tercera Edad, Jubilación.

\begin{abstract}
- Abstract
The objective of our research work is to analyze what the old ones of masculine sex make in their free time and groups of Third Age or Associations of Pensioners have been inserted in. The subjects of our search are eight old men of sixty to ninety years, users of the Maria Olinda de Castro Place, located in the district of Vila Progresso, in the city of Sorocaba. We used the qualitative investigation methodology by this to have a multidimensional character and to place the investigator in direct bonding with the investigated customer. The data collect was made by using eight semi-structured interviews, with average duration of 30 minutes each one. Also data through photos were collected. The results were divided in five categories: age, civil state, participants or not of Groups of Third Age or Associations of Pensioners, have or not freetime and whatever to desires of those old ones. We could verify that the old ones make partnerrecreational activities, with stress reduction, increase of the personal satisfaction and a ampler social interaction where sharing it is seen like something very important. Therefore, we can consider that those old ones are socialized, exerting an active paper in the community and society.
\end{abstract}

KEY WORDS: Aging, Occupational Therapy, Free Time, Third Age, Retirement 


\section{Introducción}

El objetivo de la investigación se relaciona con la exploración del tiempo libre en el anciano jubilado de sexo masculino, debido a que en la ciudad de Sorocaba se visualiza una gran cantidad de ancianos en lugares públicos, como las plazas; jugando naipes, leyendo periódicos y revistas, fumando, conversando; o sea, interactuando con personas de su edad. Sin embargo, nuestro interés principal es investigar lo que los ancianos de sexo masculino que frecuentan esas plazas realizan en su tiempo libre y si están insertados en Grupos de Tercera Edad o Asociaciones de Jubilados. Como cita Neri (1999, p. 61): ? Compartir en actividades grupales con personas de la propia generación favorece el bienestar del anciano porque facilita la emergencia de significados comunes y una mayor aproximación interpersonal...? (1) Partiendo del supuesto de que el envejecimiento de la población brasileña tuvo un rápido aumento desde la década del cincuenta, debido a la reducción de la natalidad y de la mortalidad y a los avances técnicos de la medicina y la salud publica; consideramos que envejecer envuelve varios factores como; psicológico, biológico, social, existencial, que afecta al individuo como un todo. Es un proceso individual que depende mucho del equilibrio entre las limitaciones y las potencialidades del ser humano. De acuerdo con Rosa (1996, p.90): ?Los aspectos biológicos del envejecimiento son prácticamente inevitables. Por lo tanto, el elemento fundamental que distingue a los individuos en el proceso del envejecimiento es la actitud de la persona hacia su propio envejecer. Si la persona asume una actitud saludable de aceptación de su proceso de envejecimiento, comúnmente esta aceptara esos cambios como algo natural y se ajustara mejor a esa fase de la vida. Si, por otro lado, el individuo asume una actitud de rechazo con su envejecimiento, el puede no conformarse con los cambios inevitables de su cuerpo, y eso puede, inclusive, acelerar aun más el proceso de envejecimiento.? (2 ) El anciano debe reconocer y aceptar sus limitaciones y posibilidades para tener una buena calidad de vida, pues envejecer no es dejar de vivir y aprovechar las cosas buenas de la vida, no es castrar la sexualidad del anciano, recluyéndolo en su propio mundo. Es importante saber que el placer, los deseos, el bienestar, el amor, el sexo y principalmente la felicidad, no es solo privilegio de los mas jóvenes, es derecho de todo y cualquier ser humano mientras el viva. Por lo tanto, envejecer no restringe y no niega una existencia confortable al anciano; en caso que el tenga una vivencia grupal, si no vacila en la lucha contra el conformismo y la comodidad, si no se limita en los vicios del prejuicio; descubrirá así nuevos horizontes e ideales. Conforme envejecemos, ocurren cambios en nuestras vidas, siendo una de ellas la jubilación, que viene a ser en el sentido técnico, el término de la actividad remunerada, el fin del ejercicio de una ocupación. La jubilación es uno de los momentos más importantes en la vida de la mayoría de la población anciana. Al vivir en una sociedad postmoderna, esta determinara una edad cronológica para su inicio. Sin embargo, la jubilación hace parte integral del proceso de la vida y hoy un gran número de personas vivencian esta experiencia. Esta representa un derecho común que va de acuerdo con las funciones del trabajo, del sector económico y condiciones subjetivas de cada uno. Es un momento de cambio concreto y real en la vida del individuo. Esta es la interrupción o modificación de un cierto ritmo de vida mantenido durante muchos años. Como cita Vieira (1996, p. 14): ? La jubilación es un asunto de máxima importancia en la gerontología social porque ella puede ser un hito en el proceso de envejecimiento, dependiendo de cómo cada uno la ve y siente. En su esencia esta es un derecho y no un beneficio, que debe garantizar la mantención del nivel de vida del ciudadano? (3). La manera por la cual el individuo vivirá su jubilación, estará entonces influida por su historia de vida, sus relaciones interpersonales y con la sociedad, sobre todo con el papel profesional y su modo de enfrentar las perdidas y de adaptarse a las nuevas situaciones. La jubilación debe ser vista como un proceso continuo de crecimiento y 
mejora; no debe significar el fin de la vida social, pero si como una nueva fase de la vida donde el individuo dispone de un tiempo mayor para ejecutar tareas de su interés, buscando así nuevos ideales. Sin embargo es un tiempo restante de las actividades profesionales, escolares, familiares, religiosas y políticas, donde es posible determinar libremente lo que se desea realizar, pudiendo ser una actividad de ocio, de diversión, distracción, o también una actividad de mayor significado individual y social. Para Papaleo (1996, p/ 104): ? El ocio hace parte del hacer humano, con alto significado para el desarrollo personal y colectivo de la humanidad. Necesita, por lo tanto, ser entendido en todas sus dimensiones, pues solo así llevara a la reflexión, a la crítica, al cambio, al cuestionamiento y a la transformación social.? (4) El tiempo libre es aquel en cada uno tiene para si, para hacer lo que gusta, para convivir con quien estima, o sea, para expresarse como persona. Uno de los aspectos más importante en la Terapia Ocupacional es despertar en el anciano el interés en ocupar de manera calificada y orientada su tiempo libre conquistado después de muchos años de trabajo, manteniéndolo activo, proporcionando condiciones adaptables para que el viva de forma satisfactoria y posible su día a día, pues el hacer algo en su tiempo libre puede significar a lo largo de la vida de los ancianos, formas de mantener la continuidad, la autonomía, la independencia y la elección durante el proceso de vida. Por lo tanto, el tiempo libre cuando no es llenado por una actividad, sea ella de cualquier naturaleza, puede llevar a que las personas se aíslen socialmente. Por eso existen los Grupos de Tercera Edad que desempeñan un papel fundamental en la socialización del anciano, en la recuperación de la autoestima, en la revitalización física y mental. Hay una necesidad cada vez mayor, de que los ancianos tengan, después de la jubilación, no solo una vida larga sino también rica en calidad física, mental y social. En los grupos existen programas con el objetivo de favorecer en los ancianos la restauración de una vida social, disminuyendo así los problemas de soledad, rompiendo con estereotipos y prejuicios que las personas tienden a mantener dentro de si. Esos grupos tienden a valorar a los ancianos, creando espacios para el ocio, pero también para que se valoren como ciudadanos. El grupo promueve la conquista del placer, la satisfacción, la realización de los sueños que quedaron detenidos en otras etapas de la vida, la adquisición de nuevos conocimientos y comprensión de nuevas experiencias. Partiendo de una visión holística del ser humano, tenemos entonces que la Terapia Ocupacional entiende el envejecimiento, no solamente como un momento de la vida del individuo, sino también como un proceso que envuelve modificaciones biopsicosociales, que afectan la relación del individuo con el medio, o sea, el envejecer tiene una dimensión existencial extremadamente compleja, con implicaciones tanto para la persona que la vive, como para la familia y sociedad en general. La Terapia Ocupacional en la Gerontología Social, tiene el papel fundamental de valorar el potencial existente del anciano, facilitando para el mismo la posibilidad de vivir de forma sana su proceso de envejecimiento. Es a través de la Terapia Ocupacional que podemos verificar la autonomía e independencia de los ancianos y como estos desempeñan las funciones del día a día, siempre llevando en consideración su contexto socioeconómico y cultural. La Terapia Ocupacional utiliza actividades seleccionadas para esta clientela, teniendo el propósito de estimular las áreas de autocuidado, autonomía e independencia en las AVD (Actividades de Vida Diaria ) y AVDI ( Actividades Instrumentales de la Vida Diaria ), trabajo y ocio, interviniendo en los niveles preventivos, curativos y de rehabilitación. Por lo tanto, en la Gerontología Social, la Terapia Ocupacional tiene por objetivo mantener al anciano participante y activo, integrado a la comunidad, y propiciar en el el contacto consigo mismo y con la realidad, como forma de promover su autorrealización. 


\section{Metodología}

Teniendo un concepto de la importancia de la Terapia Ocupacional, realizamos la investigación de campo, con la intención de indagar sobre lo que los ancianos jubilados de sexo masculino realizan en su tiempo libre, basándose en un enfoque cualitativo, puesto que éste ofrece contribuciones no sólo estadísticas, sino también de la expresión de sentidos y sentimientos.

Los sujetos de nuestra investigación son ancianos de sexo masculino de sesenta a noventa años, no institucionalizados. Fueron realizadas ocho entrevistas semiestructuradas. Éstas contenían preguntas centradas en el cotidiano y la ocupación del tiempo libre de los ancianos, y fueron efectuadas en un solo encuentro con una duración total de cuatro horas, en promedio treinta minutos cada una, en la Plaza Maria Olinda de Castro, localizada en la Avenida Arthur Bernardes en el Barrio Vila Progresso, en la ciudad de Sorocaba - São Paulo. Para esta pesquisa utilizamos algunos materiales como: Grabador; Cinta Magnética; Papel; Bolígrafo; Una película y una máquina fotográfica profesional.

El objetivo general fue verificar lo que los ancianos jubilados de sexo masculino realizan en su tiempo libre, si estaban insertados y cuál es su vinculación con Grupos de Tercera Edad o Asociaciones de Jubilados.

En tanto, los objetivos específicos fueron:

$\S$ Averiguar en los Grupos de Tercera Edad la cantidad de ancianos de sexo masculino;

$\S$ Conocer el "perfil" de los ancianos masculinos jubilados que frecuentaban las plazas de Sorocaba;

$\S$ Verificar cuales son las actividades de socialización del cotidiano de esos ancianos;

$\S$ Investigar cómo utilizan su tiempo libre;

$\S$ Verificar si los ancianos jubilados frecuentaban algún Grupo de Tercera Edad, o si estaban insertados en los grupos del barrio en que residen.

La plaza en que realizamos la investigación de campo posee un quiosco con mesas y bancos de hormigón, una tienda de revistas, un punto de venta de helados y jardín con árboles. Es un lugar donde existe movimiento tanto de automóviles y motos, como de personas circulando en este recorrido.

Al entrar en contacto con los sujetos de la investigación, fuimos bien recibidas por ellos, demostraron estar interesados en saber sobre nuestra formación académica y el objetivo de estar realizando este estudio. Informamos que nuestro interés era realizar una investigación de campo para el Trabajo de Conclusión del Curso de Terapia Ocupacional de la Universidad de Sorocaba.

Comentamos acerca de la función del Terapeuta Ocupacional en la Gerontología y enseguida explicamos que pretendíamos investigar el uso del tiempo libre de los ancianos jubilados que se encontraban presentes en esta plaza y si ellos formaban parte de algún Grupo de Tercera Edad. 
Los sujetos que fueron entrevistados son de sesenta a noventa años, jubilados, brasileños, la mayoría casados, no frecuentan grupos de tercera edad o asociaciones, no poseen nivel superior y utilizan su tiempo libre para dedicarse a alguna actividad de ocio como: viajes, juegos, televisión, música, bailes, entre otras.

Los ancianos lograron comprender el cuestionario, siendo en la mayoría de las veces coherentes en sus respuestas; en ningún momento hubo interferencia ni opinión de las investigadoras en las respuestas de los ancianos.

Percibimos que son personas en su mayoría extrovertidas, poseen una buena interacción con el grupo y muchos se conocen hace más de treinta años.

En su mayoría estos ancianos tienen deseos de retomar sus estudios, de aprender otras lenguas, de conocer otras ciudades de Brasil y otros países.

\section{- Resultados}

Verificamos que de las ocho entrevistas realizadas, obtuvimos algunos datos en común y otros que difieren, siendo que el $50 \%$ de los ancianos entrevistados tenían entre setenta y ochenta años y esto se debe a la mejoría de vida socioeconómica, los avances técnicos de la medicina, la salud pública y la forma en cómo los ancianos se adaptan a los factores de constitución genética, psicológica, al estilo de vida y también a las influencias de la sociedad.

Tabla 1 - Número y porcentaje de ancianos entrevistados, según la edad.

\begin{tabular}{|c|c||c|}
\hline Edad & Cantidad & \% \\
\hline \hline $60-65$ & 2 & 25 \\
\hline \hline $65-70$ & 0 & 0 \\
\hline \hline $70-75$ & 2 & 25 \\
\hline $75-80$ & 2 & 25 \\
\hline $80-85$ & 1 & 12,5 \\
\hline \hline $85-90$ & 1 & 12,5 \\
\hline TOTAL & 8 & 100 \\
\hline
\end{tabular}

Fuente: Entrevista realizada por las investigadoras el 15/06/2002

Averiguamos que el $50 \%$ de los ancianos eran casados, aunque no fue posible con nuestro instrumento de búsqueda saber si estaban en el primero, segundo o tercer matrimonio, sin embargo el hecho de estar involucrados en una relación afectiva implica en la promoción del bienestar físico y mental; aunque la soledad se torne más frecuente en esa edad, se puede envejecer sin soledad ni aislamiento. Amigos, vecinos y parientes tienen una participación importante en la vida de esos ancianos, pudiendo auxiliar en los cuidados y prevenciones de hábitos psicológicos y sociales. En cuanto a los solteros, viudos y divorciados es posible resaltar que al estar viviendo su madurez 
parece que desean encontrar una compañera para relacionarse afectuosamente, mostrando que la pasión, el amor y el sexo son posibles en cualquier edad, bastando estar abierto para este encuentro.

Tabla 2 - Número y porcentaje de ancianos entrevistados, según estado civil.

\begin{tabular}{|c|c|c|}
\hline Estado Civil & Cantidad & \% \\
\hline \hline Soltero & 1 & 12,5 \\
\hline Casado & 4 & 50 \\
\hline Viudo & 2 & 25 \\
\hline Divorciado & 1 & 12,5 \\
\hline TOTAL & 8 & 100 \\
\hline
\end{tabular}

Fuente: Entrevista realizada por las investigadoras el 15/06/2002

Otro resultado obtenido en esta entrevista es que el $62,5 \%$ de esos ancianos no participan de Grupos de Tercera Edad o Asociaciones de Jubilados, pues de acuerdo con informaciones proporcionadas vía telefónica por los coordinadores de Grupos y Asociaciones, pudimos constatar que la presencia del sexo masculino es minoría y supuestamente esto acontece debido a que los hombres no tienen la misma tendencia que la mujer a la vida social. Sin embargo, creemos que ellos se tornan más reclusos, o tienen contacto social sólo con los amigos, siendo que hay una cierta dificultad de desarrollar las actividades de los Grupos de Tercera Edad.

Aunque la cultura aún determine ciertas actividades para hombres y mujeres, las realizadas en los Grupos de Tercera Edad y Asociaciones son ofrecidas para ambos sexos.

Se cree que la cantidad de hombres en los Grupos de Tercera Edad es menor debido a algo de timidez por parte de ellos y también al recelo de asumirse dentro de la tercera edad.

Tabla 3 - Número y porcentaje de ancianos entrevistados, según participación en Grupos de Tercera Edad y Asociaciones de Jubilados.

\begin{tabular}{|c|c||c|}
\hline $\begin{array}{c}\text { Grupos de } \mathbf{3}^{\mathbf{a}} \\
\text { edad } \mathbf{y} \\
\text { asociación }\end{array}$ & Cantidad & $\mathbf{\%}$ \\
\hline Participante & 3 & 37,5 \\
\hline No Participante & 5 & 62,5 \\
\hline TOTAL & 8 & 100 \\
\hline
\end{tabular}

Fuente: Entrevista realizada por las investigadoras el 15/06/2002

Verificamos también que el $87,5 \%$ son ancianos que disponen de un tiempo libre y que en su mayoría es utilizado en estas plazas en la convivencia con los amigos y compañeros de juegos. 
Para Pekelman (1996 p.18,19), el tiempo libre empleado con calidad y satisfacción se convierte en una gran oportunidad en la vida de los ancianos, y si éste es aprovechado de manera saludable posibilita la motivación y el placer de vivir en plenitud (므).

\section{Tabla 4 - Número y porcentaje de ancianos entrevistados, que poseen o no tiempo libre.}

\begin{tabular}{|c|c|c|}
\hline Tiempo Libre & Cantidad & \% \\
\hline \hline Sí & 7 & 87,5 \\
\hline \hline No & 1 & 12,5 \\
\hline TOTAL & 8 & 100 \\
\hline
\end{tabular}

Fuente: Entrevista realizada por las investigadoras el 15/06/2002

El último resultado obtenido fue que el $37,5 \%$ de los ancianos manifestaron el interés de retomar sus estudios, pero se sienten limitados por causa de la edad. Por difícil que sea, sabemos que retomar los estudios es posible, ya que hay escuelas y universidades que pueden atender las necesidades de esta población.

Como cita Neri \& Freire In: Santos Sá \& Santos (2000); la educación es uno de los medios para vencer las barreras impuestas a los ancianos en razón de la edad y por la sociedad, promoviendo el aprendizaje de nuevos conocimientos y oportunidades para buscar el bienestar físico y emocional. Los programas educativos para los ancianos buscan atender esas necesidades, trabajando con diversos procedimientos pedagógicos, con el fin de despertar la conciencia crítica para la búsqueda del envejecimiento bien llevado. Por medio de ellos, se ha posibilitado a los ancianos la adquisición de conocimientos y participación en actividades sociales, políticas, culturales y de ocio (ㅁ).

De los ancianos entrevistados se verifica que también al $37,5 \%$ de éstos les gustaría conocer otras ciudades de Brasil y viajar a otros países.

Para Pekelman (1996), viajar es conocer un mundo nuevo. El viaje no termina con el retorno, sino permanece para siempre en la memoria de las personas que lo vivieron.

Para los ancianos, salir de la rutina del día a día puede significar una de las mejores maneras de alejar el estrés, ampliar sus horizontes y enriquecer su equipaje cultural. Para los jubilados, realizar un viaje proporcionaría también una oportunidad para relacionarse con personas diferentes a través de actividades culturales y de ocio.

Se destaca que al $25 \%$ le gustaría, si es posible, retomar su trabajo; esto se debe tal vez a la falta de adaptación a las nuevas situaciones, dejando una sensación de inutilidad, haciendo que ellos busquen alternativas para esta nueva etapa de sus vidas.

Ninguno de los ancianos entrevistados manifestó interés en pertenecer a Grupos de Tercera Edad. Consideramos que para ellos el hecho de estar en la plaza, significa estar insertado en un grupo de carácter comunitario, donde existe la posibilidad de mantener relaciones interpersonales y vivenciar actividades socio-recreativas. 
La convivencia con los demás frecuentadores de la plaza es una manera de que los ancianos establezcan buenas amistades, aprendan cosas nuevas y de pasatiempo. Es también importante que ellos utilicen el lugar para el ocio, relajándose y manteniendo relaciones socioculturales.

Muchos ancianos al salir del mercado del trabajo, tienen su red de relaciones reducida, así como también pierden el poder económico y el prestigio social que la actividad productiva proporciona en la sociedad contemporánea. El número de actividades que realizaban en relación al trabajo disminuye y el cese de este trabajo hace que los hombres tengan una necesidad de mantener el contacto familiar, buscando relacionarse con personas en la misma situación o no, buscando alternativas de nuevas actividades, como: de interacción social, ocio o aún una ocupación con fines lucrativos. Pueden también participar de Grupos de Tercera Edad y Asociaciones, o de grupos de carácter comunitario, considerando como ejemplo las plazas de los barrios de la ciudad de Sorocaba.

Con el avance de la edad, algunas veces el anciano va siendo excluido de la sociedad y comienza a sentirse parte no integrante de ésta, como si su presencia y participación no fueran necesarias para las otras personas. Así el anciano proyecta continuar siendo parte de la sociedad para, de algún modo, producir algo útil.

Sin embargo, la mayoría de los ancianos no consigue reintegrarse a la sociedad y, sin la posibilidad de ejercer alguna actividad, recurre a otras alternativas como forma de amenizar sus problemas. Luego, percibimos que hacer parte de este grupo ( Plaza ), puede funcionar como un medio de reintegración, pues posibilita, por medio de nuevas actividades como juegos y relaciones interpersonales, la búsqueda de cambios afectivos, solidaridad y diversión, entre otras. Así es que en esta plaza el anciano puede mantener sus relaciones con otras personas, realizar algún tipo de actividad, descansar y al mismo tiempo divertirse, pues es bien probable que este recinto propicie a los ancianos la sensación de ser partes activas e integrantes de la sociedad.

\section{Tabla 5 - Número y porcentaje de ancianos entrevistados, en cuanto a los proyectos futuros deseados.}

\begin{tabular}{|c|c|c|}
\hline Deseos & No & \% \\
\hline \hline Viajar & 3 & 37,5 \\
\hline $\begin{array}{c}\text { Regresar al } \\
\text { Trabajo }\end{array}$ & 2 & 25 \\
\hline $\begin{array}{c}\text { Retomar los } \\
\text { Estudios }\end{array}$ & 3 & 37,5 \\
\hline \hline TOTAL & 8 & 100 \\
\hline
\end{tabular}

Fuente: Entrevista realizada por las investigadoras el 15/06/2002 


\section{Conclusiones}

Llegamos al final de este trabajo con la sensación de que hay mucho por descubrir y relatar sobre el envejecimiento del anciano de sexo masculino y de la utilización de su tiempo libre y de la relación grupal. Sin embargo, siendo éste un estudio finito de conclusión de curso, deparamos con el hecho de tener que terminarlo, dando nuestro parecer final.

Consideramos que nuestros objetivos fueron alcanzados, pues logramos realizar la investigación y conocer sobre el tema propuesto: el tiempo libre y relaciones grupales de los ancianos jubilados de sexo masculino no institucionalizados.

En el lugar escogido para realizar nuestra investigación, fue posible comprobar nuestro dato inicial de que los ancianos de sexo masculino son la minoría en Grupos de Tercera Edad ofrecidos por instituciones, ya que se verificó en las entrevistas que sólo 37,5\% son participantes de Grupos de Tercera Edad y Asociaciones de Jubilados, siendo $62,5 \%$ no participantes.

Lo que pudimos percibir también es que ellos prefieren participar en grupos informales de carácter comunitario (en este caso en las plazas de la ciudad de Sorocaba), siendo esta opción una de las alternativas encontradas para que continúen ejerciendo su autonomía, sin dejar de vivir su papel participativo en la comunidad.

En nuestra percepción inicial creíamos que los ancianos de esta plaza, por no estar insertados en Grupos de Tercera Edad o Asociaciones de Jubilados, se encontraban ociosos y sin participación social más activa, pero a partir del momento en que realizamos nuestra investigación de campo descubrimos que esos ancianos sí desarrollan actividades ocupacionales, ayudan a sus esposas en las tareas domésticas, van al supermercado, pagan sus cuentas en el banco y utilizan su tiempo libre para realizar actividades que propicien momentos de relajamiento y placer.

También fue posible verificar en nuestra investigación que los ancianos a pesar de haber vivido la jubilación como una interrupción y cambio en su cotidiano, no presentaron sensaciones de inutilidad, porque encontraron alternativas para adaptarse a las nuevas condiciones de vida, donde ocurre la reducción de la responsabilidad del trabajo, de las preocupaciones y de los cuidados a los hijos, pasando a tener más disponibilidad de tiempo para vivir de forma saludable actividades de su interés.

En cuanto a su comportamiento, percibimos que esos ancianos se mostraron afectivos, expresivos, extrovertidos, independientes, teniendo una buena interacción grupal, pudiendo desarrollar sus papeles sin perder el liderazgo, el sentido de estar en actividad, el carácter competitivo, entre otros datos que fueron posibles observar en este grupo que investigamos.

Podemos considerarlos insertados en un grupo informal de carácter comunitario, puesto que ahí se encuentra presente, principalmente, el sentimiento de pertenecer a una parte de la sociedad.

Como cita Debert (1999, p 162): " Los programas para tercera edad fueron y están siendo creados para rescatar la dignidad del anciano, disminuir los problemas de la soledad, quebrar los prejuicios y estereotipos que los individuos tienden a internalizar. Se trata de valorar al ciudadano de más edad, creando espacios para el ocio pero 
también para " entrenamiento en el ejercicio de la ciudadanía." Son espacios para una vivencia colectiva de negación de la vejez, a través de transformación de las etapas más avanzadas de la vida en momentos adecuados a la conquista del placer, de la satisfacción, de la realización de sueños tenidos en otras etapas de la vida, de nuevos conocimientos y de la adquisición de nuevas experiencias." (ㄱ).

Fue posible verificar la utilización de su tiempo libre de forma útil y productiva, donde existe espacio para modificaciones de ideas y nuevas realizaciones.

Analizando las actividades realizadas por los ancianos en las plazas tales como: naipes, dominó y damas, percibimos que éstas propician el desarrollo en la relación interpersonal, como también a través de ejercicios mentales, ayudan a preservar la memoria, el raciocinio y la concentración, contribuyendo tanto para una maduración personal y social, como para la preservación de la salud en el transcurrir de su envejecimiento.

Finalmente, al estar insertados en grupos, en compañía con otras personas, compartiendo sus sueños y deseos, y estar próximos a los amigos, se sienten capaces de realizar alguna actividad, permitiendo el redescubrir la utilidad que poseen como y principalmente de continuar sentirse parte de algo que presente sentido, o sea, un grupo social.

Por lo tanto, podemos considerar que esos ancianos están socializados, ejerciendo un papel activo en la comunidad y en la sociedad.

Vale la pena resaltar que estudios complementarios y comparativos que pueden ser realizados tanto en otras plazas de la ciudad de Sorocaba, como en ciudades con otras características socioeconómicas, pueden traer nuevas e importantes contribuciones para la continuidad de discusiones acerca de este tema. 


\section{Referencias Bibliográficas}

1 NERI, A.L. Qualidade de vida e idade madura. 20.ed. São Paulo: Papirus, 1999; p.61.

2 ROSA, Merval. Psicologia Evolutiva 4 - psicologia da idade adulta. 80. ed. Petropólis: Vozes, 1996; p.90.

3 VIEIRA, Elaine Brandão. Manual de Gerontologia : um guia teóricoprático para profissionais, cuidadores e familiares. Rio de Janeiro : Revinter, 1996; p.14

4 NETTO, Papaléo Mateus. Gerontologia : a velhice e o envelhecimento em visão globalizada. São Paulo : Atheneu, 1996; p.104.

5 PEKELMAN, Eve. Como aproveitar melhor o tempo livre. São Paulo: Editora Gente, 1996; p.18:19.

6 NERI, A.L; FREIRE, S. A. E por falar em boa velhice. Campinas, SP: Papirus, 2000; p.93.

7 DEBERT, Guita Grin. A Reinvenção da Velhice : socialização e processos de reprivatização do envelhecimento. São Paulo: Fapesp, 1999; p.162.

8 FERREIRA, A, B, H. Minidicionário da língua portuguesa. $3^{\circ}$.ed. Rio de Janeiro, 1993. 